Bull. Mater. Sci, Vol. 4, No. 3, May 1982, pp. 321-339. (C) Printed in India.

\title{
The design of space vehicles
}

\author{
K KASTURIRANGAN \\ ISRO Satellite Centre, Bangalore 560058 , India
}

MS received 17 June 1980

\begin{abstract}
In this paper, an attempt has boen made to summarise the essential elements of a space vehicle design. After giving an overview of the methodology of spacecraft sizing and configuration, a brief outline of the technical considerations related to the design of different subsystems of the vohicle has been presented. The essential aspects related to manned systems are also discussed. Tho article concludes with the identification of some of the important payload interfaces, that are rolovant to the design of material processing experiments in space.
\end{abstract}

Keywords. Mission; satellites orbit; Salyut-6

\section{Introduction}

Near earth orbiting spacecrafts offer a unique opportunity to study those phenomena which require access to or utilisation of zero-g conditions, thereby opening a new direction of fundamental scicntific research. Exploiting this patentially ncw technique of scientific investigation represents a logical extension of the currently ongoing space programme activities. Planning of experiments related to zero- $g$ phenomena using spacecraft can be greatly aided by a general understanding of the different elements of a space vehicle design. The present paper has been written with these considerations in mind.

The design of a spacecraft system is essentially a multidisciplinary exercise that encompasses a wide spectrum of technologies. Central to the definition af such a system is the specification of a mission goal or a set of mission goals related usually to the areas of science, applications or technology. Once the mission goal is laid down, it is possible to identify a set of interactive technical elements that include on one side a spacecraft carrying a payload or a set of payloads appropriate to the mission and a compatible launch vehicle system for placing the satellite into a pre-selccted orbit and, on the other side, a network of ground-based telemetry, telecommand and tracking systems that serve to receive the data and exercise control over the spacecraft in its orbital phase. The mission normally concludes with a set of activities involving processing, analysis and interpretation of the data obtained from the experiments.

In consonance with the theme of the present workshop, in the rest of this paper, we shall address the design aspects of those of the space vehicles that are of relevance to material processing in space. Thus details of the geosynchronous 
and deep space spacecrafts have been left out of discussion. Further, in this context, it is to be noted that most of the material pracessing experiments have so far been carried out in manned missions. Accordingly, the presentation is structured such that the design considerations specific to near earth orbiting unmanned satellites are dealt with initially. Additionally, where applicable, certain special features of the design relevant to manned systems will also be touched upon. This will be followed by the description of systems unique to manned vehicles such as life-supparting systems.

The main features of the Salyut-6 space station are subsequently described to highlight the major technical elements of a manned space system. The paper concludes with the definition of a set of technical interfaces that are of interest to the planning of material processing experiments in space with such space stations. Discussions on the payload systems for the material processing have been left out since they are dealt with separately elsewhere.

\section{Orbital characteristics}

For the accomplishment of the mission goals, choice of a proper orbit for the space vehiole is often important. A variety of orbits are possible, the characteristics of which are summarised in figure 1.

For all the manned missions so far canducted, near earth orbits have been chosen in view of the nature of the goals set far such missions, the acceptable levels of radiation dosage as well as for reasons of total spacecraft weight which could be anywhere up to 80 tons as in the case of Skylab. Typically, these spacecrafts have been placed at orbital altitudes of 300 to $450 \mathrm{~km}$ and inclinations around $50^{\circ}$.

Based on the equation of motion in a central force field given by

$$
\ddot{\vec{r}}=-k\left(\vec{r} / r^{3}\right)
$$

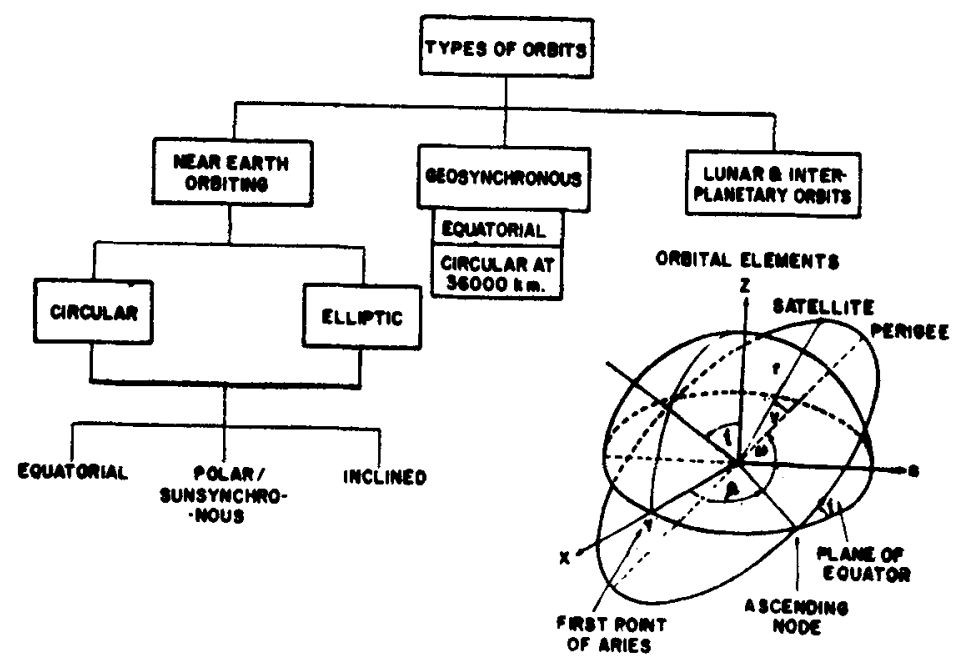

Figure 1, Orbit characteristics of space vehicles. 
the unperturbed orbit of a satellite is defined by six constants $p_{1}$ to $p_{6}$ which are introduced by the integration of this second order vectorial differential equation. Using the conventional nomenclature of classical astronomy, these six elements can be described as

$i$, the inclination-the angle between the plane of the equator and the satellite's orbital plane.

$\boldsymbol{\Omega}$, the right ascension of the ascending made-the right ascension of the point where the orbit of the satellite intersects the equatorial plane in its passage from the southern to the northern hemisphere.

$a$, the semi-major axis of the elliptical orbit.

$e$, the eccentricity of the orbit.

$\omega$, the argument of the perigee-the angle at the centre of the earth between the ascending node and the point in the orbit which is closest to the centre of the earth (perigee), and

$T$, the time of the perigee passage which is the instant when the satellite moves through the perigee point.

Oaviously, $i$ and $\Omega$ define the plane of the orbit whereas $a, e$ and $\omega$ define the size, shape and the position of the orbit in the arbital plane. Finally, the sixth element $T$ defines the position of the satellite in its orbit at this time. Of course, any other set of six independent constants, for example, the six components, $x, y, z, \dot{x}, \dot{y}, \hat{z}$ of the position and velacity vectors, appropriate to the problem, can be used as orbital elements.

A satellite, once launched into an elliptical path, with the earth's centre of gravity at one of the foci, deviates from this ideal stable orbit, fixed in the inertial reference frame of the stars, due to a variety of perturbation causes.

For the low earth orbiting satellites the two mast impartant perturbations are those arising from the earth's bulge and the atmospheric drag. The earth's bulge results in the regression of the nodes, i.e., the orbital plane of the satellite ratates slightly mare eastward on each revolution compared to what would be expected from the rotation of the earth alone. This rate of rotation of the orbital plane is given by

$$
X=9 \cdot 97(R / a)^{3 \cdot 5}\left(1-e^{2}\right)^{-2} \cos i,
$$

A second effect of the bulge is the rotation of the line apsides due to the advance of the perigee in the orbital plane. The carresponding rate $\Delta \omega$ of the advance of the perigee is given by

$$
\triangle \omega=4 \cdot 98(R / a)^{3 \cdot 5}\left(5 \cos ^{2} i-1\right)^{\%} / \mathrm{day} .
$$

For the orbits of the manned missions, these effects are in the range of 3 to $5^{\circ}$ per day. The atmospheric drag decelerates the satellite, making the arbit more nearly circular and finally causing the reentry into the atmosphere after successively reducing the altitude. For $e<0 \cdot 2$, a fairly accurate estimate of the orbit life-time can be made from the equation

$$
t_{L}=\frac{3}{4} \frac{e_{0} T_{0}}{x}
$$


where $T_{0}$ is the initial period of revolution and $x$ is the daily decrease in the period of revolution.

Coming to the manned missions, selection of orbital altitudes and inclinations beneath the Van Allen belts will best minimise the radiation hazards of the space environment. Although space processing payloads do not impose special orbit inclination requirements, an experiment using solar furnace is an exception. Such a facility would require the orientation of a focussing device towards the sun. Analysis of the orbit geometry for such a facility shows that the percentage of orbit for which the facility is in sunlight is functionally related to the altituae and the angle between the orbit normal and the sun-earth line. The maximum sun time increases as the angle between the orbit normal and the sun-earth line is minimised. To achieve $100 \%$ sunlight in a $28.5^{\circ}$ inclined orbit, typical of a Cape Kennedy launch, a minimum altitude of 930 nautical miles is required ; however, the $100 \%$ sun condition lasts only for a few arbits at a time.

\section{Overall spacecraft sizing}

To illustrate in a simple way, how a spacecraft is configured at the system level, we shall use the methodology adopted for an unmanned vehicle since the related broad principles shall be applicable to the manned vehicles also, although they are much more complex in character.

Once the orbit, the basio payload instrumentation and the mission duration are defined, it is passible ta specify the first cut power and arbit/attitude control requirements. Electrical power is needed to energise a number of onboard subsystems that include the payload, communication, instrumentation, environmental control, attitude control, guidance and even data processing. In the case of manned missions life support system requirements are additional. The attitude/orbit contral enables the praper orientation of the spacecraft commensurate with the missian needs, such as, paylaad and antenna painting, and help maintain the orbit within allowable limits.

The averall sizing of the spacecraft in respect of its weight and size is carried out at this juncture with due consideration of the constraints imposed by the launch vehicle and the environment. The canstrains from the launch vehicle side include the extent of the available volume within the shroud and the total allowable weight for achieving the desired orbit. The environmental factors include the launch stresses, the ultra-high vacuum of space and the particulate and ultraviolet radiations.

The final configuration is arrived at by a series of iterations that ensure the compatibility between the spacecraft, the rocket and the ground systems on one side and between the different subsystems of the spacecraft on the other side. Particularly if the number of subsystems of a spacecraft is $n$, then the finalisation of the canfiguration demands the identification of the related $N$ interfaces, given by

$$
N=\frac{1}{2}[n(n-1)] .
$$

Some of the major elements of this iterative procedure leading to the spacecraft configuration definition are illustrated in figure 2. 


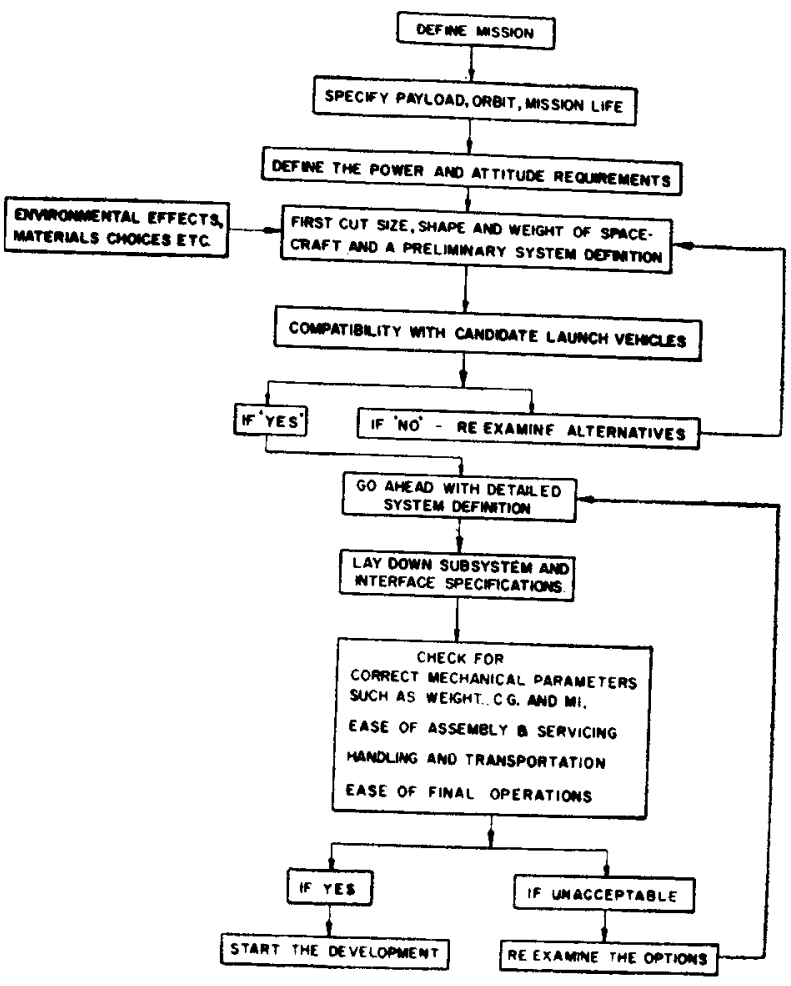

Figure 2. Sequence leading to the definition of a spacecraft.

\section{Spacecraft subsystems}

In this section, the main design considerations of some of the important subsystems of a spacecraft are given. The subsystems include structure, thermal systems, power systems, communications, control, stabilisation including attitude sensor and life-supporting systems.

\subsection{Structural subsystem}

This is the mechanical subsystem that holds together the different subsystems of the spacecraft during all the phases of the mission. The system can be broadly divided into the primary structure, the secondary structure and the appendages. Bulk of the load is supported by the primary structure and helps in the transmission of the same to the attachment paints within allowable limits. A variety of elements constitute the secondary structure that includes shields and protective covers, special or local stiffeners, damping devices, mounting bases, attachments and fasteners, etc. Elements such as antenna rods and dishes, deployable parts, solar paddles, booms, etc., normally come under the category of appendages.

Design requirements include those related to the operation prior to the flight, during powered flight and the in-orbit phase. Prior to the flight, the structure should be able to withstand the ground handling, transportation, on-stand loads, etc. During powered flight, the loads include those due to acceleration, shock, accoustic and vibration effects. In the orbit phase, the structure will encounter 
thermal stresses, micrometeoroid impacts, particle and electromagnetic radiation, eto. Further, in the case of manned spacecrafts, the structure should be able to contain artificial atmosphere, be a barrier to radiation and micrometeoroids and aid in controlling the heat balance between the vehicle and the space.

Coming to the choice of materials, the primary cnsideration is the high strengthto-weight ratio. A variety of alloys of aluminium, magnesium, titanium, beryllium and super alloys (cantaining high $\mathrm{Ni}$ and $\mathrm{Ca}$ ) have been used. Composite materials and structures employing $H_{0}$ eycomb and Sandwich constructions and using fibreglass reinforced plastics and carban reinforced plastics provide high strength-to-weight ratio. Special ceramic refractory materials or ablative materials are used for re-entry systems to withstand temperatures of the order of $4000^{\circ} \mathrm{F}$.

The validation of the structural design and the material choice is carried out by a scries of tests that are broadly classified as dynamic and static. The dynamic tests enable the evaluation of the structure under dynamic loads that include sinusoidal, random and shock type of inputs. The tests enable the study of dynamic properties such as natural frequencies, mode shapes, damping, transmissibility, dynamic stresses and deflection. The static tests simulate the steadystate accelerations. The strain levels, deformities, etc., of the structure are evaluated in these tests.

Another major element of the structural design activity is the analysis whose primary objective is to verify the adequacy of the design and to provide a prediction of the expected structural behaviour to different types of loads. The analysis encompasses aspects, such as, static analysis, dynamic studies, stability checks and further refinements. Figure 3 gives an overview of the above considerations that finally lead to the realisation of a spacecraft structure.

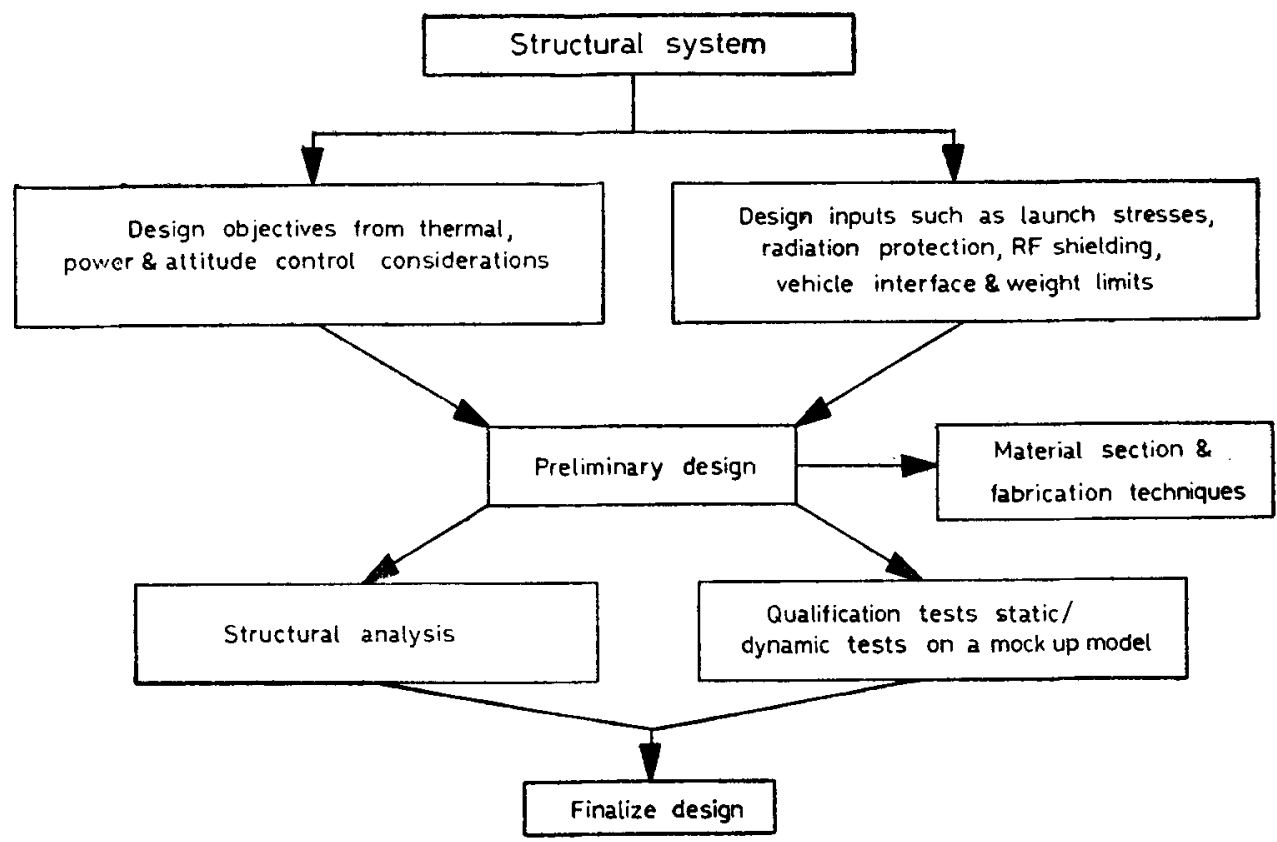

Figure 3. Design considerations of the structural subsystem. 


\subsection{Thermal control systems}

The thermal contral system of a spacecraft enables the maintenance of the temperature of the different systems within specified limits while the spacecraft is in orbit. During its normal operations in space, a near earth orbiting spacecraft receives heat inpu.t from the internal operation of the equipment, externally from sun $\left(140 \mathrm{~mW} / \mathrm{cm}^{2}\right)$, and in the form of reflected albedo radiation $\left(48 \mathrm{~mW} / \mathrm{cm}^{2}\right)$ and emission radiation $\left(23 \mathrm{~mW} / \mathrm{cm}^{2}\right)$ from earth. Further, the spacecraft radiates heat back into space which is at a temperature of about $3^{\circ} \mathrm{K}$. The objective of the thermal control design is to realise acceptable equilibrium temperatures for the different systems taking into account various heat inputs and outputs. Realisation of an effective thermal control system involves three major elements. They are design of suitable contral system devices, thermal analysis and thermal testing.

The thermal control system devices can be either passive or active. In the simplest passive case, the optical properties of the exiernal surfaces of a satellite can be suitably manipulated to control the extent of radiated or received heat. For a body, whose source of heat input is only the sun, the heat balance equation can be given by

$$
T^{4}=\left(a_{s} / \epsilon_{0}\right)\left(A_{\sigma} / A_{r}\right) \frac{S}{\sigma}
$$

where $T$ is the equilibrium temperature of the body, $a_{s}$ is the surface absorptivity for solar temperatures, $\epsilon_{0}$ is the emissivity at satellite temperatures, $A_{a}$ and $A_{\text {, }}$ are the absorbing and radiating areas respectively, $S$ is the solar constant and $\sigma$ is the Stephen-Boltzman constant. By adjusting $a_{s} / \epsilon_{0}$, using suitable paints, it is possible to realise an appropriate $T$. Another type of passive element is the multilayer insulating blankets consisting of thin alternating layers of high reflectance and low conductive materials, having conductance values of the order of $10^{-6} \mathrm{~W} / \mathrm{cm}^{\circ} \mathrm{K}$. A third type of passive element is a heat pipe that is used when heat dissipation levels are of the order of $1 \mathrm{~W} / \mathrm{cm}^{2}$ and have thermal resistance $10^{-3}$ times that of copper. Active contral elements include thermal louvers, heater mats and thermoelectric devices.

The thermal design and analysis is an interactive process of the choice of suitable thermal control elements, passive or/and active, and a preliminary analysis to check in a rough way the validity of the choices and the implementation methodology. This is followed by a detailed analysis wherein the spacecraft is divided into a number of isothermal nodes. Heat balance equations are written for all such nodes and the resulting simultaneous equations are solved by standard numerical techniques on high speed computers. Results from such an analysis help refining the implementation methodolagy.

Final validation of the thermal design is done by actual tests on a thermal mock-up model under simulated space conditions that include vacuum, temperature and solar radiation. The thermal model has the relevant thermal properties of the proposed design such as the configuration, thermal mass distribution, conductance paths, external surfaces, internal dissipation, etc.

Manned space systems have additional elements to the thermal regulation, such as, a controlled heat rejection system using a space radiator to achieve satisfactory temperature and humidity control in the cabin. 


\subsection{Power subsystem}

As mentioned earlier, the power subsystem of a spacecraft supplies power for the space vehicle subsystems that include payloads, communication, guidance and control, instrumentation and life support. The wide variety of user equipment requires power to be supplied at a variety of voltage loads, current loads and types, frequencies and degrees of regulation. This, in turn, requires a power conditioning and distribution system. There are a variety of ways by which electrical power can be generated. These include use of solar, nuclear reactor, isatopic and chemical energy. These provide the source of energy for photoelectric, electrachamical, thermoelectric, thermionics and dynamic conversion cycles. A block diagram of a typical spacecraft power supply system is shown in figure 4.

Depending on the required power level and the operating life-time, specific choices of the energy source-converter combination are possible as shown in figure 5. Fractions of a kilowatt have keen realised for unmanned missions using primary batteries for short flights and solar cells with secondary batteries for long duration orbital flighis. Fuel cells have been employed in Gemini and Apollo programmes for providing power at kilowatt levels for few days. Future manned systems, such as a manned space station may use dynamic power systems such as Brayton, Rankine or Stirling cycles.

Presently, the most widely employed power source system is the silic on solar cell panels in oonjunction with chemical batteries and/or fuel cells.

A solar cell is a thin slab of single-crystal semiconductor material, having a $p-n$ junction and work ; on the well-known photovoltaic principle of the conversion of solar radiant energy into electrical energy. The cell responsc can be estimated by

$$
Q_{0}=\int_{\lambda_{c}}^{\infty} Q_{\lambda} \eta_{\lambda} T_{\lambda} d \lambda
$$

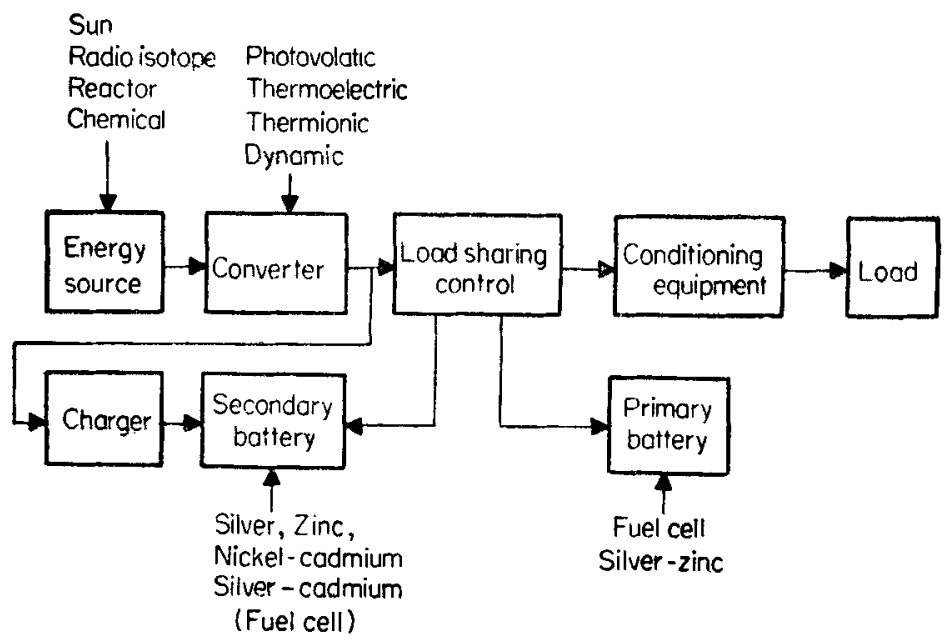

Figure 4. Block diagram of a typical spacecraft power supply system. 


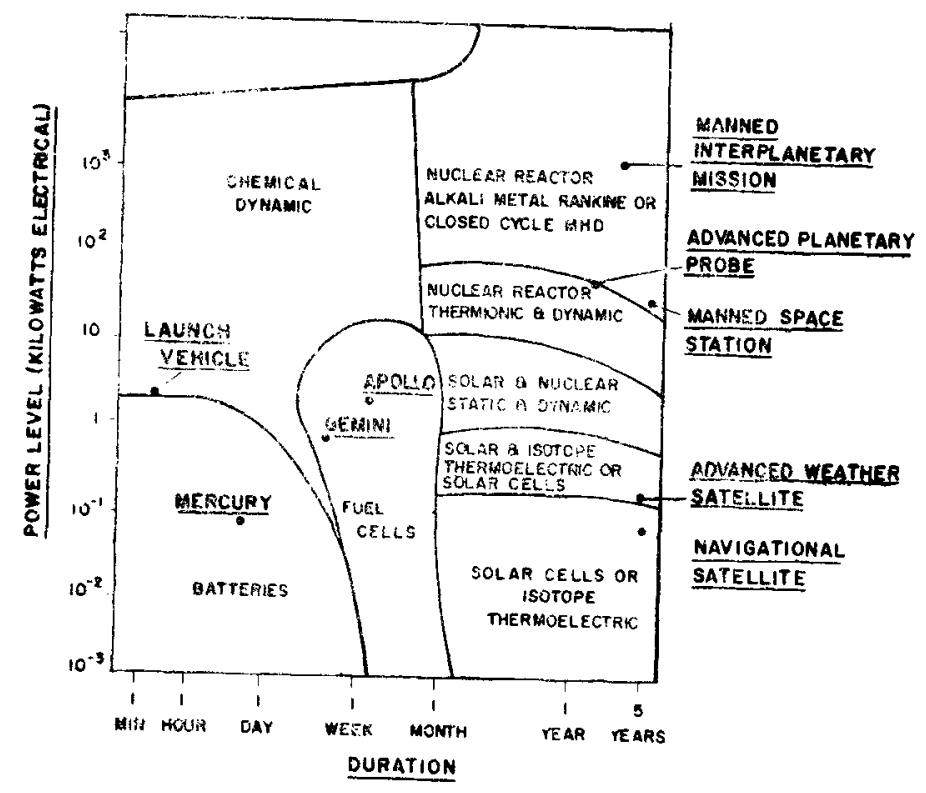

Figure 5. Role of different pawer sources for space missions.

where $Q_{0}$ is the total energy converted in the cell, $Q_{\lambda}$ is the incident power density at the wavelength interval $d \lambda, \eta_{\lambda}$ is the conversion efficiency at $\lambda$ at the interval $d \lambda$ and $T_{\lambda}$ is the transmissicn factor of the optical window at the interval $d \lambda$. Among a variety of candidates for photovoltaic materials, such as, silicon, cadmium sulphide and gallium arsenide, silicon has received the most attention. Conversion efficiency of about $15 \%$ has been realised for silicon cells. Solar panels made out of $1 \times 2 \mathrm{~cm}$ or $2 \times 2 \mathrm{~cm}$ or $2 \times 4 \mathrm{~cm}$ cells have provided power up to kilowatt levels in space mission. Panels have been either fixed to the satellite body, mounted on deployable paddles and/or oriented with respect to sun depending upon the mission.

Chemical battery takes care of the operation of the essential systems in the orbit night as well as peak load requirements and is charged in the sunlit portion of the orbit. Considering the charge-discharge cycle requirements, $\mathrm{Ni}-\mathrm{Cd}$ has been found to be the most suitible with $A g-Z_{n}$ battery as the next best. Typically $\mathrm{Ni}-\mathrm{Cd}$ battery can give $25 \mathrm{~W} \mathrm{hr} / \mathrm{kg}$.

Rest of the power system includes power conditioning and protection devices. Power conditioning systems cnable transformation of the raw power into forms suitable for the operation of various loads. The related functions include voltage regulation, frequency control and distribution. A typical power conditioning system of a spacecraft includes converters, regulators, battery charger, power control circuitry and protection devices.

\subsection{Communication system}

The communication system in a space mission performs three primary functions, namely, telemetry, telecommand and tracking. The information gathered from the experiments, various auxiliary onboard instruments as well as about the 


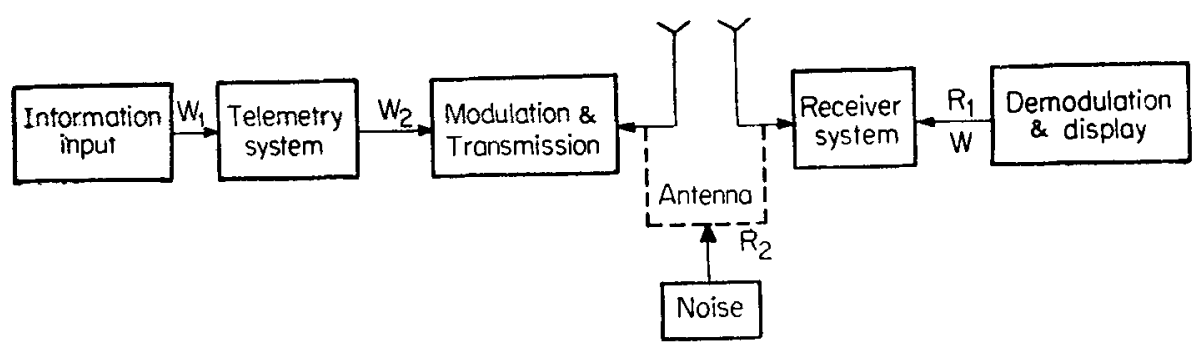

Figure 6. Elements of a typical space communication system.

spacecraft functioning itself is normally processed suitably and transmitted to a ground station via the satellite telemetry. The telecommand system enables exercising control on the spacecraft from a ground station. Operations such as putting the spacecraft in different functional modes and correcting any in-orbit anomalies are carried out using this system. The determination of the location of the satellite at different times during its orbital motion is made possible by the tracking system. The elements of a typical space communication system are given in figure 6 .

As shown in the figure, the information to be transmitted is suitably converted into voltage levels and subjected to a nonlinear operation called modulation giving a new signal-modulated carrier wave which contains the input information. This modulated carrier wave, after transmission via onboard antenna, again undergoes a nonlinear inverse transformation (demodulation) at the receiver system resulting in the retrieval of the original information. A wide spectrum of frequencies from $3 \mathrm{kHz}$ to $300 \mathrm{GHz}$ is available for the choice of carrier waves. Taking into account the ionospheric and atmospheric propagation effects, requirements of bandwidth, the available technology and a variety of similar considerations, the range $30 \mathrm{MHz}$ to $300 \mathrm{MHz}$ has been widely employed in communications for near earth satellites.

Conditioning the information signal as a part of the transmission, reception and demodulation operations results in the injection of noises with the result that the information content in the final output signal is smaller than the corresponding one carried by transmitted signal. This leads to one of the important design parameter; namely, the information handling capacity $E$ of a communication system. $E$ is given by the equation

$$
E=\frac{1_{0} W_{1} \log _{2}\left(1+R_{2}^{2}\right)}{W_{2} \log _{2}\left(1+R_{2}^{2}\right)}
$$

where $n_{c}$ is the number of information channels, $R$ and $W$ are the signal-to-noise (S/N) ratio and the bandwidth respectively as shown in figure 6 . Another important relationship of relevance to the design of a space communication is the range equation that enables the determination of the antenna size, power requirement of the carrier and the bandwidth. Far a given $\mathrm{S} / \mathrm{N}$ ratio, at a distance $R$ between the transmitter and the receiver, having antennae system gains of $G_{T}$ and $G_{R}$ respectively, the required transmitted power $P_{T}$ is given by

$$
P_{\mathrm{r}}=\frac{16 \pi^{2} R^{2} k T_{\mathrm{B}}(\mathrm{S} / \mathrm{N})}{G_{\mathrm{r}} G_{R} \lambda^{2}}
$$


where $k$ is the Boltzman's constant, $T$ is the system noise temperature of the receiver, $B$ is the transmission bandwidth and $\lambda$ is the wavelength of the carrier.

The telemetry systems are classified according to the type of multiplexing and the modulation schemes employed. Multiplexing is done either by frequency division or by time division. Modulation schemes include those invalving modulation of frequency (FM), amplitude (AM), phase (PM), pulse code (PCM), pulse amplitude (PAM) and pulse duration (PDM). Table 1 gives the comparative performance parameters of scme of the typical telemetry systems.

It is evident from table 1 that PCM-FM telemetry system has optimal characteristics in terms of $E, P_{T}$ and $W_{2}$ compared to other schemes. Further, a digital systems such as PCM has the added advantages of accuracy, ease of data storage and data precessing with computers.

Coming to the telecommand system, in a typical system known as the tone command system, the uplink carrier is amplitude-modulated with a series of audio tones, the tone sequence distinguishing the different commands. In view of the limited number of commands possible with such a system and owing to their insecure nature, tone digital commands have been used in the recent space missions. The system provides for a large number of ON/OFF commands (more than 200), real time uplink data transfer capability and memary load commands. The important design considerations for such a system include coding, choice of Hamming distance, parity chccks, bit error rate, etc. A type of coding called error correcting/error detecting code helps combating the effects of noise. The effectiveness of such an error correcting code is dictated by Hamming distance which gives the number of digits by which any of the two messages differ. For a Hamming distance of $n$, the total nu mber of errors that can be corrected is given by $(n-1) / 2$. The reliability of a message is assessed in the simplest way by parity check by counting ones or zeros and nating whether either of them is odd or even. The command system is normally designed with bit error rates better than 1 in $10^{-5}$. Once all these aspects are suitably taken care of, it is possible to realise command system with the command missing probability less than 1 in $10^{-15}$ and the probability of spurious executions less than 1 in $10^{-40}$.

Table 1. Comparative performance narameters of some telemetry systems.

\begin{tabular}{lccc}
\hline $\begin{array}{c}\text { Telemetry } \\
\text { Scheme }\end{array}$ & $\begin{array}{c}E \\
\text { (Information } \\
\text { efficiency) }\end{array}$ & $\begin{array}{c}\text { Power } \\
\text { (relative) }\end{array}$ & $\begin{array}{c}W \\
\text { (Bandwidth for } \\
\text { equal o/p infor- } \\
\text { mation capacity }\end{array}$ \\
\hline PPM-AM & 0.170 & 1 & 76 \\
FM-FM & 0.023 & 14 & 140 \\
PAM-AM & 0.073 & 250 & 18 \\
PCM-FM & 0.240 & 1.7 & 18 \\
PDM-PM & 0.036 & 11 & 110 \\
\hline
\end{tabular}


A variety of techniques are available for tracking the spacecraft. The radio techniques include tone range system to provide range, Doppler system to provide range rate and interferometric system for getting angular information of the satellite. Further, optical techniques involving cameras and laser ranging have been also employed.

\subsection{Attitude control and sensor systems}

Attitude control of a spacecraft is the orientation of the satellite fixed axes with respect to a desired reference framo, thereby providing the necessary alignment for the operation of payloads, sensors, antenna and even solar panels. The relevant information about the attitude is generated by a system of sensors that sense the directions of certain well-known landmarks in space such as the sun, the earth's magnetic field, the infrared horizon of the earth or some bright stars.

Once the design goals are laid down, there are at least four major elements to the design of a control system for a spacecraft. These include mission analysis, dynamic and reliability analysis, hardware implementation and finally testing and design evaluation.

In the mission analysis phase, the overall mission profile vis-a-vis the attitude control requirements are examined. The availability of the ground support facilities and the interfaces between the onboard and the ground systems are additional oonsiderations in the mission analysis. Further, the activities include identification of failure modes, contingency pracedures and the development of mission operational software compatible with the available computational facility.

Dynamics analysis is carried out for various control modes, ensuring stability for all possible mass distributions and flexibilities as well as disturbances such as gravity gradient, magnetic, solar radiation, aerodynamic and those arising from the operation of the onboard hardware. Stability analysis is done for all possible modes including transition modes from one phase to another.

The implementation of an attitude control system has several approaches that can be broadly classified as passive, semi-passive, active and hybrid.

The passive system controls the attitude of a spacecraft by employing elements that primarily depend on inertial tarques or external forces for their operation. The simplest method of realising a passive stabilisation is by spinning the satellite about its axis of the greatest moment of inertia (say $I_{z}$ ). The condition for spin stability is that $I_{x}, I_{y}<I_{z}$ where $I_{x}, I_{y}$ and $I_{z}$ are the moments of inertia about the principal body axes of the satellite. To damp out the undesired nutational motion arising from magnetic, gravitational, solar and aerodynamic torques, a nutation damper is usually emplayed. In another mode of passive stabilisation, the tendency of a satellite, having a large dipole moment $(\vec{M})$, to align itself along the local direction of the earth's magnetic field $\left(\vec{H}_{0}\right)$, is exploited. The torque $(\vec{T})$ on such a satellite is given by

$$
\vec{T}=\vec{M} \times \vec{H}_{0}
$$


The equation of motion, under small displacement is given by

$$
\theta=\theta_{0} \cos \left(\frac{M H_{0}}{I_{z}}\right)^{\frac{1}{2}} t
$$

and the oscillations are usually damped using permeable magnetic rods. This principle can be further exploited in semi-passive mode for any general spin axis arientation in space by installing an electromagnet along the spin axis and controlling its polarity.

A third approach is to use the gravity gradient torques arising from small, but finite, difference in the strength of the earth's gravitational field which occurs over the finite spatial extent of the satellite. A typical dumb-bell, for example, having mass $m$ at each end, separated by $2 d$ and locatcd at distance $R$ from the centre of the earth, experiences a torque given by

$$
g=3\left(G M / R^{3}\right) I \theta
$$

where $I=2 m d^{2}$ and $\theta$ is the small angle displacement. Such a torque causes the alignment of one axis of the satellite along the earth's local vertical direction.

Coming to the active control systems, there are three major types of active systems used for attitude control. In the first system called the mass expulsion system, cold gas, hydrazine or electrically-charged particles are expelled at definite velacities to produce the required control torques in the satellite. A set of 12 thrusters is normally employed, four about each axis to result in positive or negative torques. A pair of thrusters fired for short time $t$ causes the satellite to acquire an angular rate given by

$$
\triangle W=T \triangle t / I_{z}
$$

where $T$ is the torque given by the pair of thrusters. After the correction of the error, reverse direction thruster is fired such that the angu:lar velocity $\triangle W$ reduces to zero. The second approzch to active control involves the use of reaction wheel systems. The spacecraft fitted with a wheel having one axis of freedom can generate, using a coupled electrical motor, a reaction torque in either directions within the two bodies. The motor torques are so adjusted as to keep the satellite aligned with respect to the desired direction. For a complete attitude control about all the three axes, the spacecraft will require three orthogonal sets of wheels. In the third approach, gyrotorquers are used. These are constant momentum devices, a fly wheel spinning in the spacecraft at constant rpm, that can be rotated about one or two axes of the satellite through suitable gimbals. The spin axis of the wheel defining the characteristic angu.lar momentum vector can be moved within the body. As the total angular momentum of the body remains inertially fixed, the spacecraft attitude changes accordingly. A set of 3, 4 or 6 gyrotorquers can be employed to result in a suitable configuration.

The fourth aspect of the control system implementation, as mentioned earlier, is the testing and design evaluation. This involves the computer simulation of exact dynamics, disturbances and transition phase from one mode to another, hybrid simulation, using onboard hardware to the extent possible, and simulation with 3 axis gas bearing platform, using onboard hardware, sensors and reference simulators. 
The attitude sensors are used for deriving the information on orientation of the satellite axes in space for control purposes and also to interpret the data from the scientific and application experiments onboard. The attitude sensors can be electro-optical, magnetic, electromagnetic or inertial. The electro-optical sensors work on the principle of detecting the signals, either visible or infrared, radiated or reflected from sun, moon, stars, earth, planets, etc. For example, the sun sensors, either digital or analog, using silic on photodetectors or detector arrays have typically an accuracy of about half a degree. Thase that track the geometrical centre of sun have accuracies of a few are seconds. Star sensors are used where high accuracy attitude determination is needed, since they are the most accurate sources of direction in space. Star sensors can be either of mapping type or tracking type. Star mappers are used in spinning satellites where the sensor scans the sky and gives a series of coded signals corresponding to different stars. Using this data in conjunction with a star map the attitude can be determined. Star trackers are usually used in three axis stabilised satellites. A small region of the sky is imaged and is scanned using electromechanical scanners or electronic scanning. The stars in this region are identified and attitude information is obtained in two axes. Infrated horizon sensors, working on the principle of detecting the IR radiation from earth corresponding to $\mathrm{CO}_{2}$ band (around 15 microns), are also employed as satellite attitude sensors. The accuracy that can be realised using these IR horizon sensors is generally 0.03 to 0.01 degree.

Magnetic sensors measure the geomagnetic fiald alcng three orthogonal axes of the satellite. The attitude is computed by knowing the direction of the field in orbit. Flux-gate magnetometers are generally used as sensors.

Among the ineltial sensors the most impartant one is the rate integrating gyro. Gyroscopes perform their usual function of detecting rates relative to inertial space. The total torque $\overrightarrow{\boldsymbol{G}}$ on the gimbal is (see figure 7 for notations)-

$$
\vec{G}=\vec{\omega} \times \vec{h}
$$

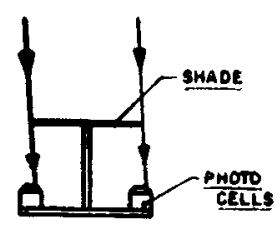

1.SUW SENSOR

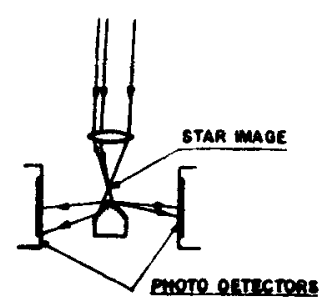

3. STATKC-SPUT STAR TRACKER

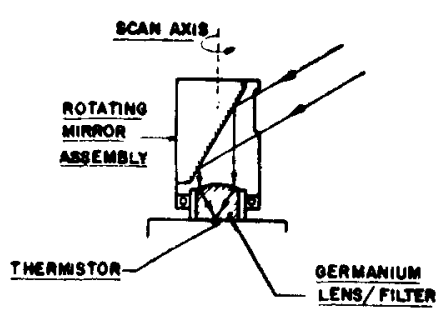

2. HORIZON SCANMER

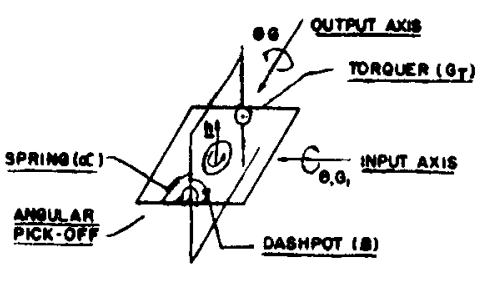

4. SINGLE-AXIS OYRO

Figure 7. Attitude sensors used onboard spacecrafts. 
where $\vec{h}$ is the spin angular momentum and $\vec{\omega}$ is the gimbal total angular velocity. If the gyro is rotated about the input axis and $a=0$, we have a torque resisting the rotation

$$
\boldsymbol{G}_{i}=h \dot{\theta_{G}}=h^{2} \dot{\theta} \mid \boldsymbol{\beta}
$$

since the relation for integrating rate gyro is obtained with $a=G_{T}=0$ and is

$$
h \theta=\boldsymbol{\beta} \theta_{\mathrm{G}} \text {. }
$$

Of some impartance is the use of a roll rate gyrascope for yaw measurement in an earth pointing satellite. If the direction of the yaw axis is controlled to the local vertical, using say a horizon sensor, then in the presence of a yaw error, a rall rate gyro will detect a component of orbital angular velocity.

Besides the attitude control systems described above, some spacecraft carry propulsive systems for periodic orbit adjustments, as in the case of earth resources and surveillance missions, and for dearbiting before re-entry as in the case of manned missions.

\subsection{Life support systems}

The basic requirement of the life support system is to provide a controlled and physiologically acceptable environment for the crew during all phases of a mission which may include launch, orbital flight, entry and post landng. The system must provide for a pressurised shirt sleeve envircnment, for pressu re suit operation during normal and emergency conditions and for food, water and waste management functions. The system should also disperse the electronic equipment heet loads to keep the environment temperature within habitable limits. The parameters that constitute the principal design criteria are mission duration, operational modes, crew size, cabin total pressu re, cabin leakage rates, power level of electrical and electronic equipment located in the cabin and type and weight of secondary power system. Crew metabolic rates and environmental requirements are also important determinants in defining the life support system. A typical range of metabolic values for normal spacecraft operations is given in table 2.

Table 2. Some metabolic parameters for normal manned spacecrafts.

\section{Lb/man-day}

Metabolic oxygen consumption

$$
\begin{aligned}
& 1 \cdot 4-2 \cdot 2 \\
& 1 \cdot 6-2 \cdot 7 \\
& 5 \cdot 0-8 \cdot 0 \\
& 2 \cdot 8-5 \cdot 0 \\
& 1 \cdot 1-1 \cdot 9 \\
& 3 \cdot 0-20 \cdot 0
\end{aligned}
$$

Urine production

Food (dry ashless basis)

Wash water 
Representative space cabin environmental specifications are given in table 3 . Basic life support systems include those for atmospheric supply (including storage methods), $\mathrm{CO}_{2}$ removal and reduction, water and waste management and trace contaminant control. Details of these systems are available in the references given at the end of this paper.

\section{Development of spacecraft hardware}

Orbiting of any spacecraft being a one shot affair, the related hardware devclopment methodolo.gy involves careful design, fabrication, testing and analysis. The final model for flight is the result of a variety of inputs derived fiom the initial fabrication and testing of a number of models such as mochanical, thermal and electrical models, each for the verification of its particular performance criteria. The mechanical model evaluation already outlined under structural system ensures the ability of the final hardware to withstand launch stresses, ground handling and orbital environment. The thermal model tested under the simulated space conditions verifies the efficacy of the proposed thermal control approach. Electrical model enables the verification of electrical specification of the different subsystems and the payloads as well as their interface compatibility.

The inputs from the three models cited above form the basis for the fabrication of a prototype model which is tested to the full level corresponding to the environments to be encountered in the launch and the orbital phases of the mission. This model thus serves as the final design verification unit, before embarking on the fabrication of the flight model. The flight madel is tested only to the extent necessary to identify fabrication/component deficiencies. It is to be also mentioned that both proto and flight models use high reliability components that have been subjected to strict quality control during the manufacturing processes. Figure 8 summarises the sequence leading to the development of a flightworthy satellite that is finally launched into the orbit.

\section{Salyut-6 space station}

Salyut-6 is a manned orbiting space station used for a variety of experiments in science, application and technology by the Soviet Union. This station which operates in conjunction with one or two Soyuz spacecrafts that ferry cosmonauts

Table 3. Space cabin environmental specifications.

\begin{tabular}{ll}
\hline Oxygen partial pressure & $3 \cdot 1-5 \cdot 0$ psia \\
Diluent & Nitrogen or helium \\
Talerable $\mathrm{CO}_{2}$ concentration & $3 \cdot 8-7 \cdot 6 \mathrm{~mm} \mathrm{Hg}$ \\
Relative humidity & $30-70 \%$ \\
Cabin temperature & $70 \pm 10^{\circ} \mathrm{F}$ \\
\hline
\end{tabular}




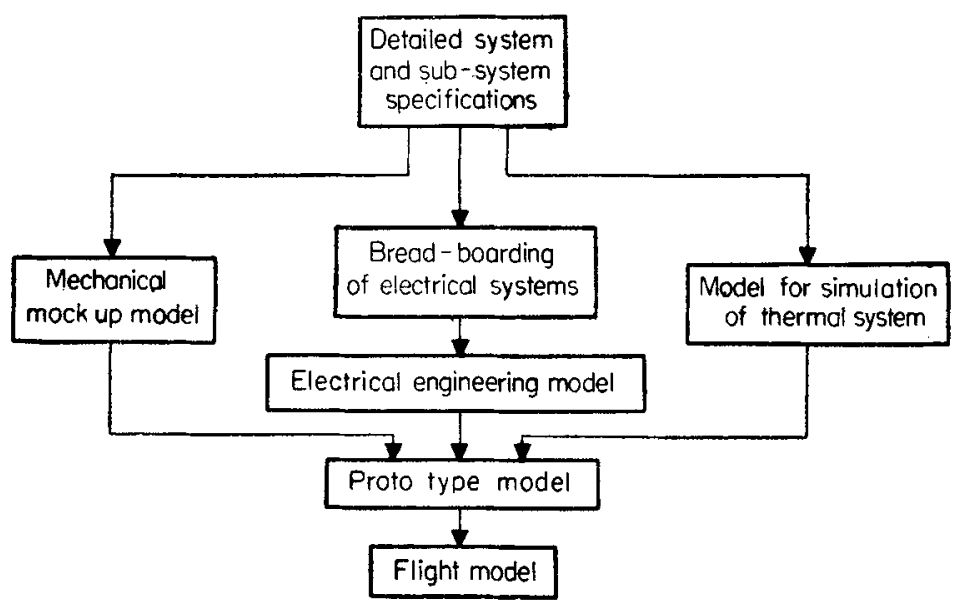

Figure 8. Sequence leading to the development of spacecraft hardware.

to the station, orbits at a near circular orbit of about $300 \mathrm{~km}$ and an inclination of $51^{\circ}$. Salyut-6 with two Soyuz spacecraft weights about 32 tons and measures 30 metres in length. Typically, it can carry 2 cosmonauts and can go up to a maximum of 6 cosmonant. The volume of the station alone is about $90 \mathrm{M}^{3}$.

Salyut-6 is powered by solar batteries of total area $60 \mathrm{M}^{\mathfrak{z}}$ and can produce up to $4 \mathrm{~kW}$ of power. The telemetry system of the station returns 2000 bits of data per second related to scientific and experimental functions. Thermal regulation and attitude control systems are installed in their final form on the station. The orientation of the station user a mathematical electronic memary and two sets of sensors evaluating heat and infrared radiation from the earth, thus determining the pasition of the station with respect to the earth's surface. Ion sensors, measuring the flow of 'ionic wind', make it possible to derive the aricntation of the station with respect to the direction of flight. Further, gyroscopes are also employed as attitude sensors.

The Delta navigation system onbcard Salyut-6 uses a computer which continually reads parameters from the radio altimeter and radial velocity meter. It has an accuracy of position lacation of the order of 2 to $3 \mathrm{~km}$ in longitude and latitude and up to several hundred metres in altitude.

The life support system on-board besides having the usual elements mentioned earlier, additionally has a water regeneration system, recovering liquid from a condensor, providing drinking water for the crew. The system ccllccts moisture from the air, separates it from the gas mixture, purifies and decontaminates it and then mineralises it.

The interior colouring of soft pestel colours for the cabin provides a psychological effect of a homely atmosphere for the cosmonauts.

The auxiliary spacecrafts, namely Soyuz and Progress carry out the functions of ferrying cosmonauts, cargo, fuel and other needs of maintaining and operating the station in orbit. With the regular refuelling missions of the Progress series of spacecraft, the station can remain in use uptc five years.

Salyut-6 contains two tonnes of equipment for scientific research in biology, astronomy, earth resources survey, smelting of metals, etc. To illustrate the 
type of material processing experiments carried out onboard Salyut-6, the main features of Splav-01 ano Kristall experiments are discussed below.

The Splav-01 experiment has three heat chambers called hot, cold and gradient areas. The hot area is maintained at a temperature of $1100^{\circ} \mathrm{C}$ and is controlled to within $5^{\circ}$ of the required value by a computer. The furnace weighs $23 \mathrm{~kg}$ and uses $300 \mathrm{~W}$ when operating. Molybdenum reflectors inside the furnace concentrate the heat into the sample and ensure that the temperature of the furnace wall does not exceed $40^{\circ} \mathrm{C}$. The outside portion of the furnace is connected via a small airlock to outer space to aid heat rejection and to enable the melting to occur in vacuum. Samples are contained in capsules, each containing three crystal ampules that fuse when subjected to heating. Monocrystals are formed in the gradient area and three-dimensional crystallisation is achieved in the hot and cold sections of the furnace. The Krystall kiln is used to obtain semiconductor materials by the zone melting techniques and also has been used to fuse optical glass.

\section{Payload interface}

In this section, certain payload interfaces of relevance to the planning of a material processing experiment onboard a spacecraft are mentioned. In this context, it is of interest to know the $g$ levels, inside a near earth orbiting vehicle, caused due to a variety of perturbations that produce corresponding accelerations. These are summarised in table 4 . These perturbations limit the minimum achievable $g$ values. Further, figure 9 gives the results of a study of the gravity field as a function of position in a near earth orbiting manned spacecraft such as Apollo.

There are at least five major interface elements that have to be considered in system engineering the payload. These include physical characteristics, resources required, environmental factors, mission factors and the electromagnetic compatibility. The fhysical characteristics are the weight, volume and shape of the payload. Resources include the specification of size and skill of the crew, average and peak electrical power, data handling needs, heat rejection as well as contral panel and display requirements. A variety of environmental factors are to be considered which include acceleration, shock, vibration, etc., during launch and re-entry, and temperature conditions in the orbit phase. Safety considerations,

Table 4. Some factors limiting in-orbit $g$ levels achievable in near earth satellites.

\begin{tabular}{ll}
\hline \multicolumn{1}{c}{ Accelerations } & \multicolumn{1}{c}{ In-orbit $g$ level } \\
\hline Orbital drag (200 NM) & $3.3 \times 10^{-8}$ to $3.8 \times 10^{-7}$ \\
Large thruster (900 Lbf) & $1.8 \times 10^{-2}$ to $4.7 \times 10^{-2}$ \\
Vernier thruster (25 lbf) & $3.0 \times 10^{-4}$ \\
Gravity gradient & $1 \times 10^{-6}$ \\
\hline
\end{tabular}




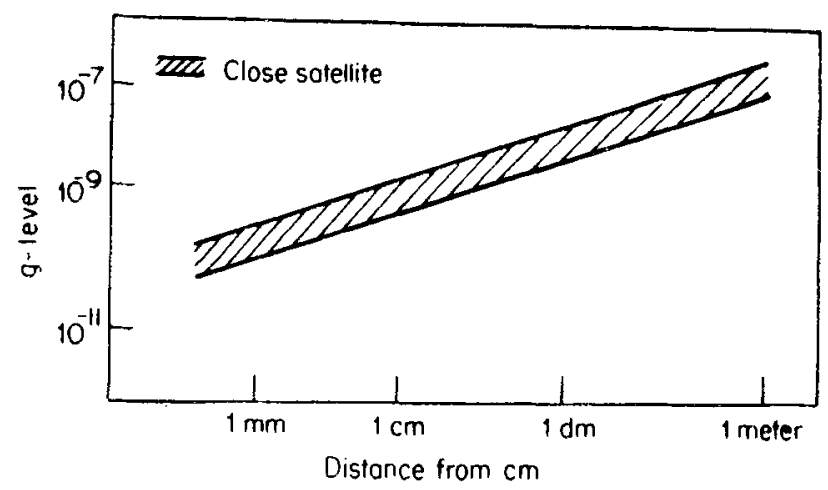

Figure 9. $g$ magnitude as a function of distance from the centre of mass of a typical low orbiting satellite.

pre-launch access, orbit characteristics, etc., come under the category of mission factors. The electromagnetic compatibility specifying the susceptability of the spacecraft systems to conducted and radiative electromagnetic emission from the payload and vice versa is another important payload interface criterion.

\section{Acknowledgements}

The author wishes to express his gratitude to Prof. U R Rao, Director, ISRO Satellite Centre, Bangalore, for his interest in this work. Financial support came from the Department of Space, Government of India.

\section{References}

Abraham L H 1966 Space technology-spacecraft systems, NASA SP-65

Adams J L 1966 Space technology-spacecraft mechanical engineering, NASA SP-66

Badgley P C (ed.) 1965 Scientific experiments for manned orbital flight AAS Sci. Technol. Ser. 4 p. 261 (Washington: Am. Astronaut Soc.)

Singer F (ed) 1964 Torques and attitude sensing in earth satellite (New York : Academic Press) Groves G V (ed.) 1965 Dynamics of rockets and satellites (Amsterdam: North Holland)

Heitchue Jr. R D (ed.) 1968 Space systems technology (New York: Reinhold Book Corporation)

Kidger N 1971 Spacefight 21178

King-Hale 1960 Satellites and Scientific Research (London: Routledge and Kegan Paul)

Scull J R 1966 Space technology-spacecraft guidance and control, NASA SP-68

Taylor K R, Hammel R L, Mock P R, Smith A G and Stevenson R D 1976 Materials science in space, Proceedings II European Symp., Itali, ESA Spl. Publ. 